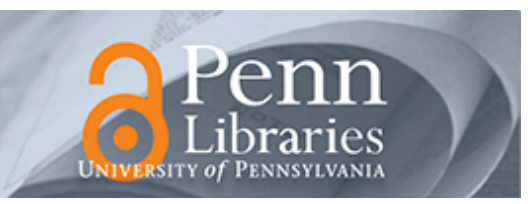

University of Pennsylvania

ScholarlyCommons

June 2005

\title{
A Leg Configuration Measurement System for Full-Body Pose Estimates in a Hexapod Robot
}

\author{
Pei-Chun Lin \\ University of Michigan \\ Haldun Komsuoglu \\ University of Michigan \\ Daniel E. Koditschek \\ University of Pennsylvania, kod@seas.upenn.edu
}

Follow this and additional works at: https://repository.upenn.edu/ese_papers

\section{Recommended Citation \\ Pei-Chun Lin, Haldun Komsuoglu, and Daniel E. Koditschek, "A Leg Configuration Measurement System for Full-Body Pose Estimates in a Hexapod Robot", . June 2005.}

Copyright 2005 IEEE. Reprinted from IEEE Transactions on Robotics, Volume 21, Issue 3, June 2005, pages 411-422.

This material is posted here with permission of the IEEE. Such permission of the IEEE does not in any way imply IEEE endorsement of any of the University of Pennsylvania's products or services. Internal or personal use of this material is permitted. However, permission to reprint/republish this material for advertising or promotional purposes or for creating new collective works for resale or redistribution must be obtained from the IEEE by writing to pubs-permissions@ieee.org. By choosing to view this document, you agree to all provisions of the copyright laws protecting it.

This paper is posted at ScholarlyCommons. https://repository.upenn.edu/ese_papers/321

For more information, please contact repository@pobox.upenn.edu. 


\title{
A Leg Configuration Measurement System for Full-Body Pose Estimates in a Hexapod Robot
}

\author{
Abstract \\ We report on a continuous-time rigid-body pose estimator for a walking hexapod robot. Assuming at least \\ three legs remain in ground contact at all times, our algorithm uses the outputs of six leg-configuration \\ sensor models together with a priori knowledge of the ground and robot kinematics to compute \\ instantaneous estimates of the 6-degrees-of-freedom (6-DOF) body pose. We implement this estimation \\ procedure on the robot RHex by means of a novel sensory system incorporating a model relating \\ compliant leg member strain to leg configuration delivered to the onboard CPU over a customized cheap \\ high-performance local wireless network. We evaluate the performance of this algorithm at widely varying \\ body speeds and over dramatically different ground conditions by means of a 6-DOF vision-based ground- \\ truth measurement system (GTMS). We also compare the odometry performance to that of sensorless \\ schemes-both legged as well as on a wheeled version of the robot-using GTMS measurements of \\ elapsed distance.

\section{Keywords} \\ body pose estimation, hexapod robot, leg configuration, legged locomotion sensing, legged odometry, \\ robot proprioception, strain-based sensor

\section{Comments} \\ Copyright 2005 IEEE. Reprinted from IEEE Transactions on Robotics, Volume 21, Issue 3, June 2005, \\ pages 411-422. \\ This material is posted here with permission of the IEEE. Such permission of the IEEE does not in any way \\ imply IEEE endorsement of any of the University of Pennsylvania's products or services. Internal or \\ personal use of this material is permitted. However, permission to reprint/republish this material for \\ advertising or promotional purposes or for creating new collective works for resale or redistribution must \\ be obtained from the IEEE by writing to pubs-permissions@ieee.org. By choosing to view this document, \\ you agree to all provisions of the copyright laws protecting it.
}




\title{
A Leg Configuration Measurement System for Full-Body Pose Estimates in a Hexapod Robot
}

\author{
Pei-Chun Lin, Student Member, IEEE, Haldun Komsuoḡlu, Member, IEEE, and Daniel E. Koditschek, Fellow, IEEE
}

\begin{abstract}
We report on a continuous-time rigid-body pose estimator for a walking hexapod robot. Assuming at least three legs remain in ground contact at all times, our algorithm uses the outputs of six leg-configuration sensor models together with a priori knowledge of the ground and robot kinematics to compute instantaneous estimates of the 6-degrees-of-freedom (6-DOF) body pose. We implement this estimation procedure on the robot RHex by means of a novel sensory system incorporating a model relating compliant leg member strain to leg configuration delivered to the onboard CPU over a customized cheap high-performance local wireless network. We evaluate the performance of this algorithm at widely varying body speeds and over dramatically different ground conditions by means of a 6-DOF vision-based ground-truth measurement system (GTMS). We also compare the odometry performance to that of sensorless schemes-both legged as well as on a wheeled version of the robot-using GTMS measurements of elapsed distance.
\end{abstract}

Index Terms-Body pose estimation, hexapod robot, leg configuration sensor, legged locomotion sensing, legged odometry, robot proprioception, strain-based sensor.

\section{INTRODUCTION}

$\mathbf{T}$ HE HEXAPOD RHex [1] exhibits unprecedented mobility for a legged autonomous robot [2]. Using an open-loop feedforward control strategy, the machine runs at speeds exceeding five body lengths per second on even terrain [3] and negotiates badly broken and unstable surfaces, as well as stairs [4]-[6].

In our initial studies with sensor-based controllers, we have observed significant behavioral improvement from simply computed feedback corrections arising from cheap and inaccurate sensory devices [7], [8]. Theoretical and simulation evidence [9] suggests that, with full-body state estimates available throughout the stance and flight phases of locomotion, considerably greater agility should be forthcoming. Thus motivated, we have extended our initial work on RHex leg configuration models [10] to develop a leg-based rigid-body pose estimation algorithm [11] for a hexapod robot assuming a gait with no aerial phase. This represents an important first step in a general full-body state estimator we are presently developing by fusing other inertial sensor-based data.

Manuscript received January 27, 2004; revised June 3, 2004. This paper was recommended for publication by Associate Editor J. Angeles and Editor F. Park upon evaluation of the reviewers' comments. The work was supported in part by the DARPA/SPAWAR Contract N66001-00-C-8026 and Contract N66001-03-C-8045.

P.-C. Lin is with the Department of Mechanical Engineering, University of Michigan, Ann Arbor, MI 48105 USA (e-mail: pclin@umich.edu).

H. Komsuoglu and D. E. Koditschek are with the Department of Electrical Engineering and Computer Science, University of Michigan, Ann Arbor, MI 48105 USA (e-mail: hkosmuog@umich.edu; kod@umich.edu).

Digital Object Identifier 10.1109/TRO.2004.840898
Notwithstanding its importance for a more agile and responsive RHex [9], we have been surprised to discover there is no prior account of a complete body pose sensor in the mobile robot literature. ${ }^{1}$ By pose, we mean the full 6-degree-of-freedom (DOF) rigid body coordinates-3 DOF describing center of mass (COM) translation (the so-called positioning problem), and 3 DOF describing the orientation of the body-relative to a fixed inertial frame. Of course, the positioning problem has been treated extensively for wheeled vehicles where the traditional dead-reckoning approach combines odometry and gyro data [12], [13] to obtain high-quality estimates of local translation. More recent research is concerned with the fusion of exteroceptive (vision [14], ultrasonic [15], or GPS [16]) sensor data for purposes of obtaining precise global positioning by periodically recalibrating the accumulated dead-reckoning error. In contrast, body orientation estimation has been investigated in the legged robot literature for high-DOF bipeds [17], [18], whose stability and balance must be actively controlled by state feedback. ${ }^{2}$ There is some prior work on exteroceptive (vision based) [20]-[22] approaches to position estimation for legged machines but no account of the legged odometry problem. Thus, our treatment of complete pose appears to be novel. Determining the relative pose of a manipulated object has won significant attention within the robotic hand literature. However, like most legged robots, the majority of robot hands and grippers are composed of rigid links whose configuration measurement is carried out by traditional joint position sensors. Whereas strain is traditionally used for force [23], [24] or torque [25] measurement, we will derive an empirical pose model from strain.

It is intuitively clear that knowledge of the configuration relative to the body of each leg in contact with the ground, together with information about the ground contact points yields complete pose information. We begin by detailing this calculation in Section II-a matter of standard geometry and linear algebra involving the position of the presumably known contact-toes in body coordinates. For traditional successively jointed rigid link legs, such leg configuration information can be computed from joint space sensors using traditional kinematic models. For a robot like RHex which has only one actuator per leg and

\footnotetext{
${ }^{1}$ We conducted systematic searches for mobile robot state estimation(estimator), mobile robot odometry, mobile robot pose, legged robot positioning(odometry), legged robot pose(estimation), across the standard bibliographic databases-Engineering Index (Compendex), IEEE Xplore, and ISI Web of Knowledge.

${ }^{2}$ We have found one paper concerned with orientation estimation for a multilegged robot [19] based upon a more traditional inertial measurement unit (IMU) sensor suite (gyro and inclinometers). Since that scheme has never been implemented on a physical robot (and we are unable to do so, lacking for the present on our test platform, RHex, the sensor suite it presumes), we cannot compare the algorithms' relative performance.
} 
relies on passive mechanical compliance as an intrinsic component of its mobility, traditional kinematic models can be replaced by empirical models, as we show in Section III. Finally, in Section IV, we report on the accuracy of the resulting estimator, both as a source of within-stride body pose information as well as integrated legged odometry over varying surface conditions and at different speeds. We also compare the performance to that of sensorless schemes-both legged as well as on a wheeled version of the robot. In Section V, we conclude by reviewing briefly the prospects for extended full-body state estimation arising from a fusion of this novel sensor with more traditional rate gyro and accelerometer outputs.

\section{Computation OF Body Pose}

This section introduces a computational algorithm for continuous measurement of full body pose for a hexapod robot. We will focus on alternating tripod walking because of its familiarity and importance in RHex, but the computations below generalize to a family of gaits characterized by two conditions: 1) the body is supported by at least three legs with noncollinear toes at any given time and 2) ground contact legs have no toe slippage. $^{3}$

In an alternating tripod walking gait, we identify two intervals: the single-stance phase 4 and the double-stance phase when all legs are in ground contact. This suggests a hierarchically structured algorithm with two levels: 1) a low level, discussed in Section II-A, operating during individual single-stance phases computing the body pose in a locally defined coordinate system, that we will term the tripod coordinate system $\mathcal{T}$, rigidly related to the world coordinate system $\mathcal{W}$ and 2) a high-level sequence of compositions, described in Section II-B, relating the tripod coordinate systems in consecutive single-stance phases to evaluate the body pose with respect to the world coordinate system $\mathcal{W}$.

\section{A. Pose Computation During Single Stance}

Assume a leg model $\mathbf{s}_{i}\left(z_{i}\right)$ for each toe of the support triangle $i=1,2,3$, where $z_{i}$ denotes the sensory measurements available regarding the configuration of the kinematic chain connecting the robot body to the $i$ th toe and $\mathrm{s}_{i}$ represents the point of toe contact with respect to the robot body coordinate system $\mathcal{B}$. For example, in the RHex implementation to be detailed below in Section III-B, $z_{i}$ introduced in (4) consists of the kinematic parameters relating the $i$ th hip frame to the body $\gamma_{i}$, together with the strain across the compliant portion of the leg as read from the sensor suite $\sigma_{i}$.

With the definition of the support triangle $\mathcal{S}$, shown in Fig. 1 whose vertices are identified with the noncollinear toes of the ground contact legs, we can derive the unit vectors along the two edges of the support triangle $\mathcal{S}$ that intersect at $\mathbf{s}_{1}$ as

$$
\mathbf{e}_{1}:=\frac{\mathbf{s}_{2}-\mathbf{s}_{1}}{\left\|\mathbf{s}_{2}-\mathbf{s}_{1}\right\|_{2}} \quad \mathbf{e}_{2}:=\frac{\mathbf{s}_{3}-\mathbf{s}_{1}}{\left\|\mathbf{s}_{3}-\mathbf{s}_{1}\right\|_{2}}
$$

\footnotetext{
${ }^{3}$ These conditions guarantee that the toe contacts yield a well-defined coordinate system fixed in the world frame. Appropriate generalizations of the calculations would extend the computation of full body pose to other kinds of legged robots, like quadrupeds or even bipeds with foot (surface) contact.

${ }^{4}$ Single-stance phase denotes the instance when the body is supported by only one tripod where the three toes of the front and rear ipsilateral legs and the middle contralateral leg of a tripod are all in contact with the ground, as depicted in Fig. 1.
}

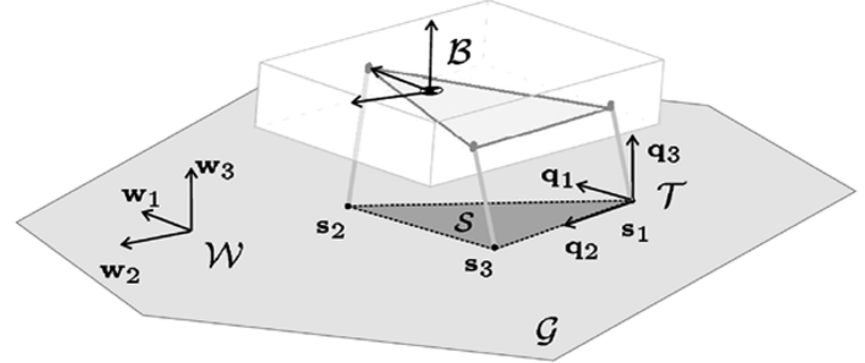

Fig. 1. Sketch illustrating the robot on a flat ground plane $\mathcal{G}$ within a single-stance phase during which it is supported by only three legs whose toes define the support triangle $\mathcal{S}$. Attached to the support triangle, we define a tripod coordinate system $\mathcal{T}$.

Now define the tripod coordinate system $\mathcal{T}$ with origin at $\mathbf{s}_{1}$ and orthonormal basis derived by Gram-Schmidt Orthogonalization as

$$
\mathbf{q}_{1}:=\frac{\mathbf{e}_{1}-\left(\mathbf{q}_{2}^{T} \mathbf{e}_{1}\right) \mathbf{q}_{2}}{\left\|\mathbf{e}_{1}-\left(\mathbf{q}_{2}^{T} \mathbf{e}_{1}\right) \mathbf{q}_{2}\right\|_{2}} \quad \mathbf{q}_{2}:=\mathbf{e}_{2} \quad \mathbf{q}_{3}:=\mathbf{q}_{1} \times \mathbf{q}_{2}
$$

represented in the body coordinate system $\mathcal{B}$. Then a rigid transformation $\mathbf{h}$ relates points $\mathbf{b}$, expressed in the body coordinate system, $\mathcal{B}$, to points expressed in the tripod coordinate system, $\mathcal{T}$, by

$$
\mathbf{h}(\mathbf{b}):=\mathbf{B}\left(\mathbf{b}-\mathbf{s}_{1}\right)
$$

where $\mathbf{B}:=\left[\begin{array}{lll}\mathbf{q}_{1} & \mathbf{q}_{2} & \mathbf{q}_{3}\end{array}\right]^{T}$.

\section{B. Leg-Based Odometry Via Composition of Single Stance Measurements}

We use the term odometry to denote the computation of instantaneous body pose in world coordinate system $\mathcal{W}$ relative to a frame originally aligned with that of the body before the initiation of its motion. We will now detail how the single-stance phase pose computations described above, in Section II-A, can be integrated over multiple steps to generate this continuous computation of absolute body pose.

First, compute the homogeneous transformation between the tripod coordinate systems of consecutive single-stance phases $\mathcal{T}_{j}$ and $\mathcal{T}_{j+1}$ as follows. Assuming that the toes defining these tripod coordinate systems are stationary (there is no slippage and no liftoff) throughout their presumed stances, and assuming that there is an adequate period of double support (the two stance phases overlap for a time sufficient to complete their respective single-stance pose computations), both coordinate systems are related to the same (moving) body coordinate system $\mathcal{B}$. Now, assuming that the prior tripod coordinate system $\mathcal{T}_{j}$ has been expressed in world coordinates, the representation of its successor $\mathcal{T}_{j+1}$ in the world coordinate system follows by the properties of rigid transformations in a straightforward manner that we now detail.

We index single-stance phases $j=0,1, \ldots$ according to their occurrences over the course of locomotion where we denote the $j$ th single stance tripod coordinate system by $\mathcal{T}_{j}$. Without loss of generality, assume that the tripod coordinate system of the first single-stance phase coincides with the world coordinate system $\mathcal{T}_{0} \equiv \mathcal{W}$. 


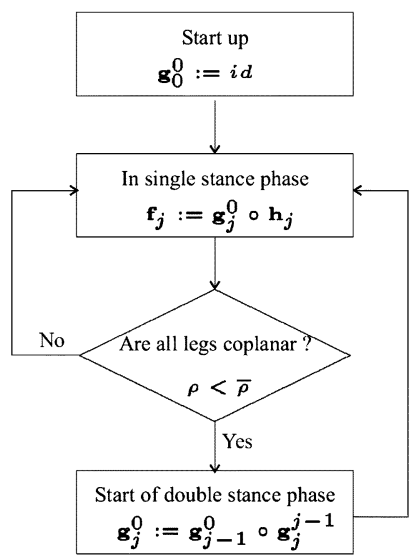

(a)

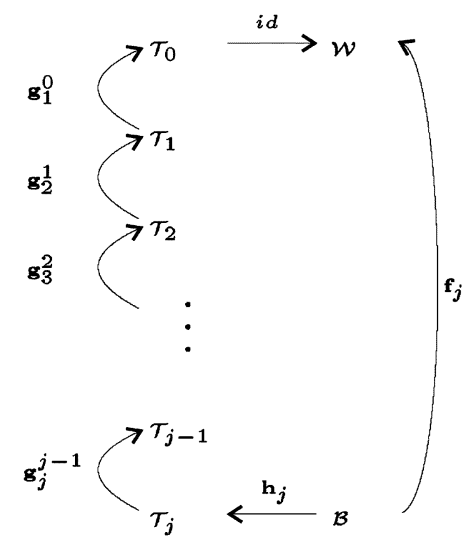

(b)
Fig. 2. (a) Flowchart for the within-stride body pose computation and leg odometry functions. (b) Commutative diagram relating the tripod coordinate systems $\mathcal{T}_{j}$.

The key for establishing the relationship between the tripod coordinate systems of two consecutive single-stance phases is the fact that there exists an intermediate double-stance phase where all legs are in ground contact. This allows us to define simultaneously the tripod coordinate systems of the single stance phase before $\mathcal{T}_{j-1}$ and after $\mathcal{T}_{j}$ this double-stance phase. Letting $\mathbf{h}_{j}$ denote the map from the body coordinate system $\mathcal{B}$ to the $j$ th tripod coordinate system $\mathcal{T}_{j}$, defined in (1), we compute the map relating the two tripod coordinate systems of consecutive single-stance phases $\mathrm{g}_{j}^{j-1}: \mathcal{T}_{j} \rightarrow \mathcal{T}_{j-1}$, given by

$$
\mathrm{g}_{j}^{j-1}:=\mathbf{h}_{j-1} \circ \mathbf{h}_{j}^{-1}
$$

In the online implementation of the pose computation algorithm, we need to detect a double-stance phase in order to compute the transformations between tripod coordinate systems of consecutive single-stance phases $\mathbf{g}_{j}^{j-1}$. Taking advantage of the flat ground plane $\mathcal{G}$ and presuming that all toes lie on the same plane only during the double-stance phase, in our application we empirically set a threshold $\bar{\rho}$ on a planarity function $\rho(\mathbf{s})$, detailed in Appendix I to determine the double-stance phase. Fig. 2(a) illustrates the flow diagram of the complete online body pose computation algorithm utilizing the planarity function $\rho(\mathbf{s})$.

The commutative diagram of Fig. 2(b) illustrates the sequential relationship between the tripod coordinate systems $\mathcal{T}_{j}$. As a direct consequence, the map $\mathrm{g}_{j}^{0}: \mathcal{T}_{j} \rightarrow \mathcal{W}$, relating the $j$ th tripod coordinate system $\mathcal{T}_{j}$ to the world coordinate system $\mathcal{W} \equiv \mathcal{T}_{0}$, can be defined recursively as

$$
\mathrm{g}_{j}^{0}:=\mathrm{g}_{j-1}^{0} \circ \mathrm{g}_{j}^{j-1}, \quad j \in\{1,2, \ldots\}
$$

where $\mathbf{g}_{0}^{0}:=i d$. This in turn leads to the definition of the rigid transformation $\mathbf{f}_{j}:=\mathbf{g}_{j}^{0} \circ \mathbf{h}_{j}$ that relates points $\mathbf{b}$, expressed in the body coordinate system $\mathcal{B}$ during the $j$ th single-stance phase, to their representation in the world coordinate system $\mathcal{W}$, which we prefer to write as

$$
\mathbf{f}_{j}(\mathbf{b}):=\mathbf{A}_{j}\left(\mathbf{b}-\mathbf{c}_{j}\right)
$$

The body pose is now read off of the entries of the transformation matrices in the familiar manner. The COM translation in lateral $(x)$ and fore/aft $(y)$ directions as well as body orientation in yaw $(\gamma)$ are computed from rigid transformation $\mathbf{f}$, shown in (2) in the standard manner [26] because these three configuration variables require the whole history of locomotion. Taking advantage of the flat ground plane $\mathcal{G}$, the COM translation in the vertical $(z)$ direction as well as body orientation in pitch $(\alpha)$ and roll $(\beta)$ are computed from the within-stride rigid transformation h, shown in (1) in the standard manner [26] to reduce the possible accumulation error.

\section{Short Discussion}

The algorithm is quite general, remaining valid, in principle, on any terrain respecting which a fixed toe coordinate system can be established. ${ }^{5}$ In practice, two caveats mitigate against its utility on badly broken or unsteady ground. Considering the latter, for sufficiently treacherous terrain, the toe frame will cease to be statically stable, and slipping legs will invalidate the assumptions upon which the computation is based. In these settings, the ability of a legged machine like RHex to balance dynamically will exceed the ability of this quasi-static strain-based model to sustain accurate pose; leg strain data is likelier to be more useful as a means of determining ground reaction forces; pose information would require the IMU we discuss in the paper's conclusion. Considering the former, the value of this algorithm in isolation also seems likely to diminish on badly broken ground-even terrain that affords good stable toe holds-since the orientation of the resulting toe frame relative to one fixed in the static world will presumably be completely unknown. Again, we anticipate that the addition of IMU-based data (for example, knowledge of the direction of gravity) will prove vital in such settings. Along these same lines, the reader should note that there is no intrinsic need for a tripod of ground contacts-merely a statically stable toe frame as might even be established by a biped's single foot.

\section{Strain-BAsed MeAsurement of Leg Configuration}

The essential novelty of the RHex design arises from its reliance upon unactuated leg compliance in place of powered joints [1]. During stance, its body and ground contact legs form a closed kinematic chain whereby the pose of the body is algebraically related to the kinematic configurations of the ground contact legs. This section concerns a novel sensor for measurement of the kinematic configuration of four-bar legs under realistic operating conditions.

We introduce the four-bar mechanism and its sensory infrastructure in Section III-A, model it kinematically in Section III-B, detail the model identification procedure in Section III-C, and validate the fitted model in Section III-D.

${ }^{5}$ Note, however, on uneven terrain, that the simple planarity measure would need to be replaced by touchdown information for each leg to afford detection of a double stance. Moreover, as we discuss below, the presumption of a known fixed toe coordinate frame may be unrealistic in the absence of some additional exteroceptive sensor to provide the absolute (world frame) location of each toe position when on uneven ground. 

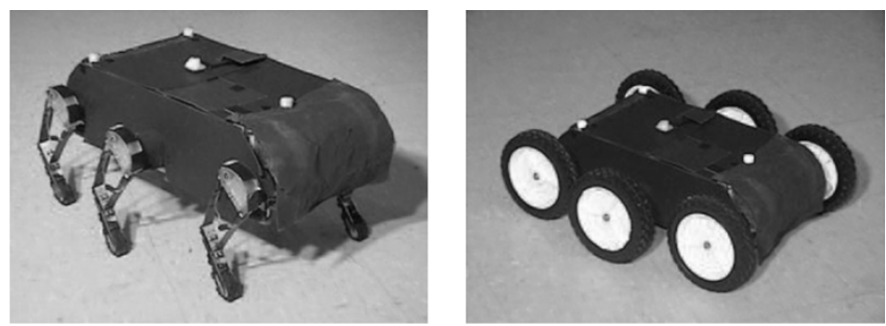

(a)

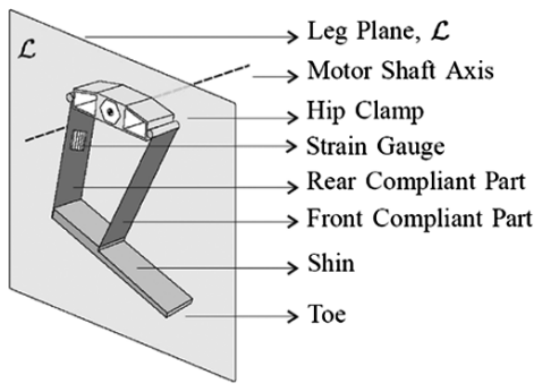

(b)

Fig. 3. (a) RHex (with four-bar leg) and wheeled RHex. (b) Sketch of the four-bar leg mechanism with its key components.

\section{A. Four-Bar Leg Mechanism}

The RHex four-bar leg [27] illustrated in Fig. 3(b) is a passive mechanical system composed of four parts: a hip clamp, front compliant member, rear compliant member, and a shin. The compliant members which are thin rectangular fiber glass strips are rigidly fixed at one end to the shin and connected to the hip clamp at the opposite end by a revolute joint (hinge) creating a four-bar linkage structure effectively operating within a two-dimensional (2-D) leg plane denoted by $\mathcal{L}=\mathbb{R}^{2}$. The hip clamp rigidly attaches this leg structure to the hip motor shaft such that the shaft axis is normal to the leg plane $\mathcal{L}$ and goes through its origin.

The leg compliance is designed to be soft in the radial direction within the leg plane $\mathcal{L}$, but extremely stiff in the other two directions, as can be verified by direct measurements. ${ }^{6}$ Our empirical study of Section III-C also indicates that the locus of the leg's physical toe positions during the normal walking operation of present interest lies in a very thin set within the leg plane $\mathcal{L}$. Thus, we adopt the simplifying assumption that a four-bar leg is effectively a 1-DOF mechanism whose configuration may be measured by a single strain gauge. The reader should note that this 1-DOF simplification accommodates an experimental observed fact regarding this particular leg design rather than any restriction in the sensing approach. Considerations of space and convenience preclude our mention here of subsequent work on alternative RHex legs [28] that turn out to operate in a large open subset of the leg plane. Sensing their configuration incurs two strain measurements but yields similar end results to those reported here.

We choose to utilize strain across the rear compliant member, $\sigma \in \Sigma:=\mathbb{R}^{+}$, for computing the configuration of this 1-DOF

${ }^{6}$ The high stiffness in the tangential direction within the leg plane is due to the difficulty to perform the compliant parts in high energy buckling mode, and that in the third direction results from four-bar geometry and a high moment of inertia.

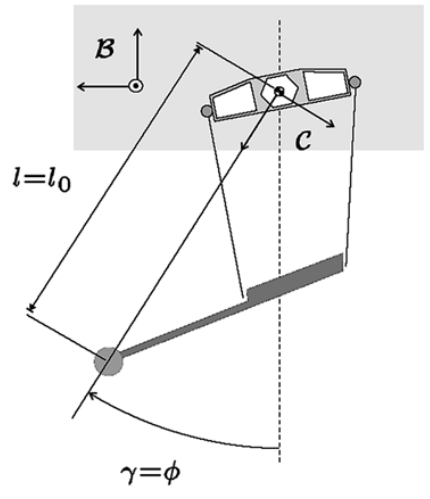

(a)

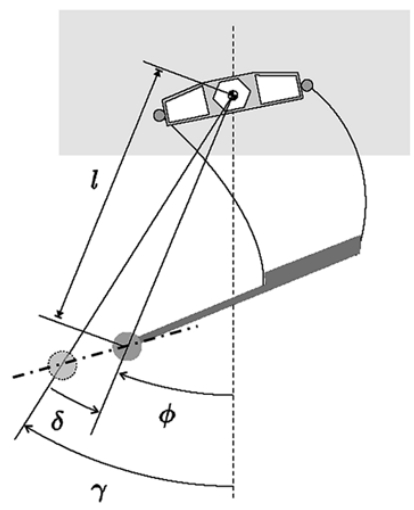

(b)
Fig. 4. Four-bar leg kinematic sketch: (a) unloaded and (b) during compression. The dashed curve represents the approximate locus of physically relevant toe positions.

leg. A strain gauge (Vishay EA-250BK-10C) is installed near the hinge-connecting rear compliant member to the hip clamp where the strain $\sigma$ is the smallest. In our empirical studies, the maximum strain measured during tripod walking gait is approximately 1000 microstrain which falls within the $10^{6}$ cycle at 1500 microstrain range reported in the strain gauge specifications. ${ }^{7}$

RHex's freely rotating legs introduce a significant technical difficulty in taking measurements from sensors installed on them-this is essentially a remote sensing problem. In our implementation, strain gauge measurements are transferred to the PC on robot body over a bi-directional multinode wireless communication network, LegNet [29], featuring a 50-kbaud communication channel over a $916-\mathrm{MHz}$ carrier connecting a master located in the PC104 stack on the body to six self-contained microcontroller-based slave units mounted on each leg. In this setting, we achieve synchronous sampling of all strain gauges at $333 \mathrm{~Hz}$ at 6-b resolution.

\section{B. A Kinematic Leg Model for Four-Bar Legs}

We represent the kinematic configuration of the four-bar leg by the location of its toe with respect to its clamp part. For this purpose, we define the polar clamp coordinate system $\mathcal{C}:=$ $\mathbb{R}^{+} \times S^{1}$, whose configuration variables include: the distance between the hip attachment point and the toe $l \in \mathbb{R}^{+}$or the effective leg length; and the angular deflection from the rest position of the toe $\delta \in S^{1}$, as depicted in Fig. 4(b).

Adoption of a first-principles model relating flexible leg member strain to leg configuration would necessitate an elastic model for laminate fiberglass, its realization for the specific materials used in the four-bar, followed by a derivation of the resulting kinematic functional form for these legs. Given the large dynamic range of strain in typical operation, the consequently limited resolution of the strain gauges due to wireless transmission motivates recourse to a far simpler phenomenological model-treating this as a system identification problem

${ }^{7}$ At a typical walking speed, the legs recirculate at roughly $2 \mathrm{~Hz}$, hence we should expect mechanical sensor failures at a specified leg every $5 \times 10^{5} \mathrm{~s}$, hence, with six operating simultaneously, at some leg every $8 \times 10^{4} \mathrm{~s}$. Thus, at a typical a cruising speed of $1 \mathrm{~m} / \mathrm{s}$, we would expect to travel $80 \mathrm{~km}$ before suffering a leg sensor failure. 
where the leg is an unknown mechanical compliance with input strain and output leg configuration, using standard data-driven techniques to fit the parameters. In any case, because the leg mass is a negligible fraction of the robot's body, we choose to ignore damping, effective inertia, and other dynamical features of this 1-DOF passive mechanism.

Pursuing this phenomenological approach, we assume that the force-extension relation in the legs can be characterized by a scalar potential function and choose to model its gradient- the function relating strain $\sigma \in \Sigma$ to toe position $\mathbf{u} \in \mathcal{C}$ - using a polynomial $\mathbf{m}: \Sigma \rightarrow \mathcal{C}$

$$
\mathbf{u}=\left[\begin{array}{l}
l \\
\delta
\end{array}\right]=\mathbf{m}(\sigma):=\left[\begin{array}{l}
\sum_{i=0}^{N}\left[a_{i} \sigma^{i}\right] \\
\sum_{i=0}^{N}\left[b_{i} \sigma^{i}\right]
\end{array}\right]
$$

where $N$ is the number of data points, and the length coefficients $\left\{a_{i} \mid i=0, \ldots, N\right\}$ and deflection coefficients $\left\{b_{i} \mid i=\right.$ $0, \ldots, N\}$ are computed by the model parameter identification in Section III-C.

Each hip clamp coordinate system $\mathcal{C}_{i}$ is related to the body coordinate system $\mathcal{B}$ by a homogeneous transformation $\mathbf{n}_{i}^{\gamma_{i}}$ : $\mathcal{C}_{i} \rightarrow \mathcal{B}$, which is parameterized by the angular position of hip shaft $\gamma_{i}$. Combining this with leg configuration model $\mathbf{u}_{i}=$ $\mathbf{m}_{i}\left(\sigma_{i}\right)$, it directly follows that the toe position of the $i$ th leg in the body coordinate frame $\mathcal{B}$ is given by

$$
\mathbf{s}_{i}\left(\gamma_{i}, \sigma_{i}\right)=\mathbf{n}_{i}^{\gamma_{i}} \circ \mathbf{m}_{i}\left(\sigma_{i}\right), \quad i=1,2, \ldots, 6 .
$$

\section{Model Parameter Identification}

To determine the coefficients in (3) assuming a ground contact leg with no toe slippage, we utilize the benchtop setup described in Appendix II, yielding a database whose entries consist of three measurements: 1) the strain in the back compliant member $\sigma ; 2)$ the displacement of the toe from its rest position $(\delta x, \delta y)$; and 3$)$ the components of the ground reaction force at the toe $\left(F_{n}, F_{t}\right)$. Due to manual leg manipulation, the raw benchtop data set has a nonuniform distribution incorporating regions of the leg plane $\mathcal{L}$ that are rarely if ever visited during normal walking. Therefore, the first step in the system identification procedure will be data cleaning, described in Appendix III, to generate relatively uniform distribution of data points as well as pruning away those not physically relevant during normal robot operation.

For each point in the resulting clean data set, we compute the leg configuration variables $(l, \delta)$ and plot them individually against the strain readings as shown in Fig. 5. Coefficients of the leg model in (3) are computed by standard ordinary least squares. Fig. 5 exhibits typical linear fits.

Letting $\hat{\mathbf{d}}$ denote model prediction for a stream of $M$ physical measurements $\mathbf{d}=\left(d_{1}, \ldots, d_{M}\right)$, taken from a leg, where $d_{i}$ denotes either length $l_{i}$ or deflection $\delta_{i}$, we measure model performance by the percentage normalized rms error $\xi(\mathbf{d}, \hat{\mathbf{d}})$ between the original data $\mathbf{d}$, and the corresponding model output $\hat{\mathbf{d}}$, given by

$$
\xi(\mathbf{d}, \hat{\mathbf{d}}):=\frac{1}{d_{\max }} \sqrt{\left(\|\mathbf{d}-\hat{\mathbf{d}}\|_{2}^{2} / M\right)} \times 100
$$

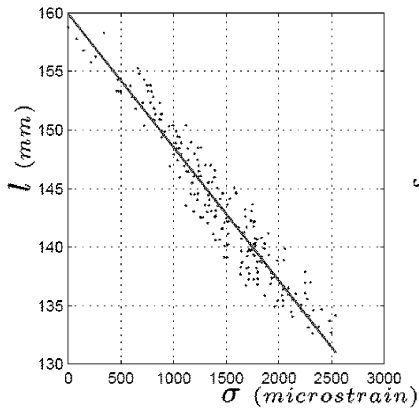

(a)

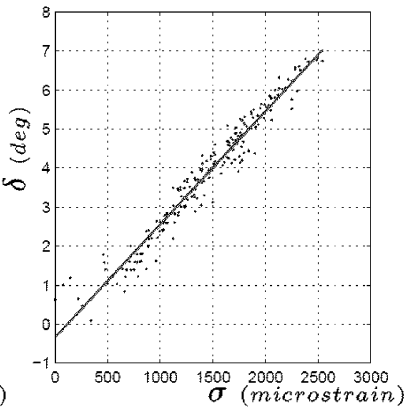

(b)
Fig. 5. Linear leg model. (a) Leg length model. (b) Leg deflection angle model with physically relevant data.

TABLE I

PERCENTAGe RMS ERROR FOR Kinematic Leg Models of VARIOUS POLYNOMIAL DEGREE

\begin{tabular}{c|c|c|c|c}
\hline Polynomial & \multicolumn{2}{|c|}{ Model Fit } & \multicolumn{2}{c}{ Cross Validation } \\
Degree & $\xi(l, \hat{l})$ & $\xi(\delta, \hat{\delta})$ & $\xi(l, \hat{l})$ & $\xi(\delta, \hat{\delta})$ \\
\hline \hline 1 & $1.12 \%$ & $4.87 \%$ & $1.20 \%$ & $5.44 \%$ \\
\hline 2 & $1.11 \%$ & $4.79 \%$ & $1.16 \%$ & $5.51 \%$ \\
\hline 3 & $1.05 \%$ & $4.53 \%$ & $1.11 \%$ & $5.19 \%$ \\
\hline 4 & $1.05 \%$ & $4.43 \%$ & $1.11 \%$ & $5.26 \%$ \\
\hline
\end{tabular}

where $M$ is the length of the data vector and $d_{\max }$ is the maximum value in original data.

Table I summarizes the fitting and cross validation ${ }^{8}$ performances of the leg model in (3) with different polynomial order $N$, where small values indicate successful model prediction. Since the improvement in model performance is insignificant for quadratic and higher order polynomials, we choose to use the linear model due to its simplicity. Fig. 5 presents the resulting linear fits for the leg length $l$ and deflection angle $\delta$, respectively.

\section{Verification of Leg Model}

In order to assess the performance of the kinematic leg model in (3) under realistic operating conditions, we ran a set of experiments where the output of the model is compared against the leg configuration as measured by a visual test station described in Appendix IV.

Different walking speeds are associated with different leg compression patterns, resulting in different ranges of strain as well as in different dynamic behavior. Thus, variation of speed emerges as the most important of physical effects over which to challenge the validity of this model. Table II exhibits the data collected with mean (avg) and standard deviation (std) from two sets of five runs at two different speeds: slow speed $(0.25 \mathrm{~m} / \mathrm{s})$ and fast speed $(0.51 \mathrm{~m} / \mathrm{s})$, where the performance of the model in each case is evaluated by percentage normalized rms error, defined in (5), between ground truth measurement and model output. Fig. 6 shows the comparison of these plots for two typical runs from slow and fast speeds.

\footnotetext{
${ }^{8}$ This term denotes our simple estimates of the predictive accuracy of these models, as follows. In each data set, we selected at random a small amount of data (typically $20 \%$ of the available input output pairs) for use as a cross-validation test population. The models were fit to the $80 \%$ unselected population and then used to "predict" the input output relationships within the test population.
} 

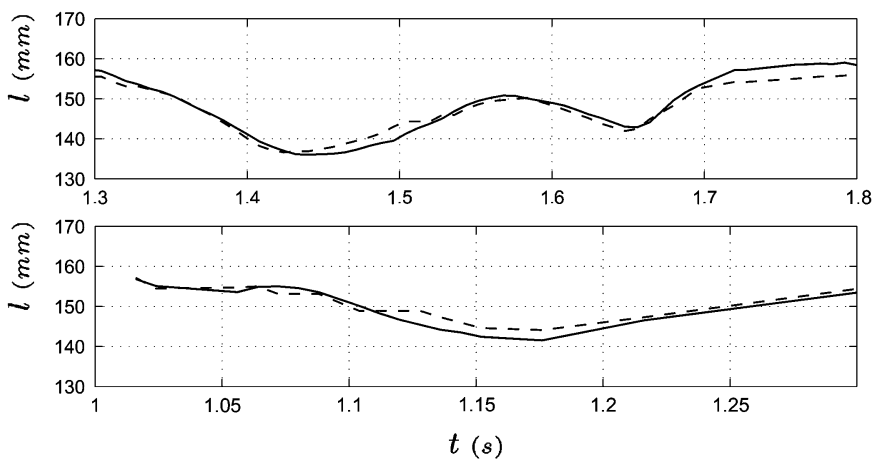

(a)
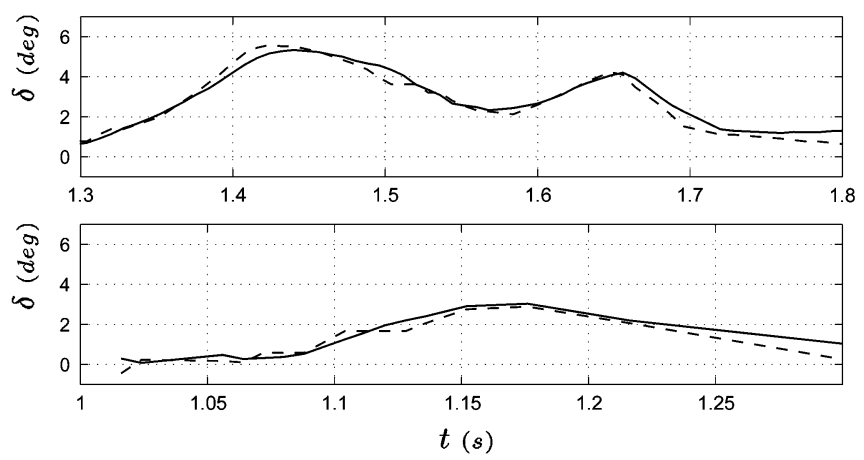

(b)

Fig. 6. Comparison of ground truth (solid line) and model based (dashed line) leg kinematic configuration at $0.25 \mathrm{~m} / \mathrm{s}$ (top) and $0.51 \mathrm{~m} / \mathrm{s}$ (bottom). Plots for (a) the effective leg length $l$ and (b) deflection angle $\delta$.

TABLE II

Performance Summary: Kinematic Leg Configuration Model at VARYiNG SPEEDS AS COMPARED TO GROUND TRUTH VisuAL MEASUREMENT

\begin{tabular}{c|c|c|c|c}
\hline Exp \# & \multicolumn{2}{|c|}{$0.2 \mathrm{~m} / \mathrm{sec}$} & \multicolumn{2}{c}{$0.45 \mathrm{~m} / \mathrm{sec}$} \\
& $\xi(l, \hat{l})$ & $\xi(\delta, \hat{\delta})$ & $\xi(l, \hat{l})$ & $\xi(\delta, \hat{\delta})$ \\
& $(\%)$ & $(\%)$ & $(\%)$ & $(\%)$ \\
\hline \hline Exp \#1 & 1.29 & 10.34 & 1.25 & 15.00 \\
\hline Exp \#2 & 1.19 & 7.77 & 1.11 & 16.95 \\
\hline Exp \#3 & 1.29 & 8.77 & 1.12 & 12.01 \\
\hline Exp \#4 & 1.14 & 6.38 & 1.11 & 12.97 \\
\hline Exp \#5 & 1.29 & 8.89 & 1.23 & 9.61 \\
\hline avg & 1.24 & 8.43 & 1.16 & 13.31 \\
\hline std & 0.06 & 1.31 & 0.06 & 2.51 \\
\hline
\end{tabular}

We observe very small error in effective length $l$, which remains less than $2 \%$ of the rest length independent of the walking speed. The error of deflection angle seems to grow as the speed increases with an apparent upper bound at $17 \%$. Although the percentage error in deflection angle $\delta$ is not small, its absolute value (below $5^{\circ}$ ) turns out to have negligible adverse effect upon the pose estimator, as reported below.

\section{PERFORMANCE}

We now report on the performance of the pose estimation scheme presented in Section II using the strain-based leg sensor introduced above in Section III. We investigate performance under realistic operating conditions over multiple steps, reporting on the effects of increasing speed and decreasing surface friction. The reader should note that RHex's relatively constrained kinematics preclude the exercise of its yaw degree of freedom when it walks with no aerial phase and no toe slippage, hence the implementation we discuss in this section will entail no data of that nature-we discuss measurements involving only the five configuration variables: the displacement of the center of mass in the lateral direction $x$, forward direction $y$, and vertical direction $z$, as well as orientations given by pitch $\alpha$ and roll $\beta$ in the world coordinate system $\mathcal{W}$.

In our assessment, we compare the output of the computational algorithm to independent measurements made by a visual ground-truth measurement system (GTMS) described in Appendix V, different from the one used in the previous section described in Appendix IV. Performance is measured by the vector rms error between the outputs of the algorithm
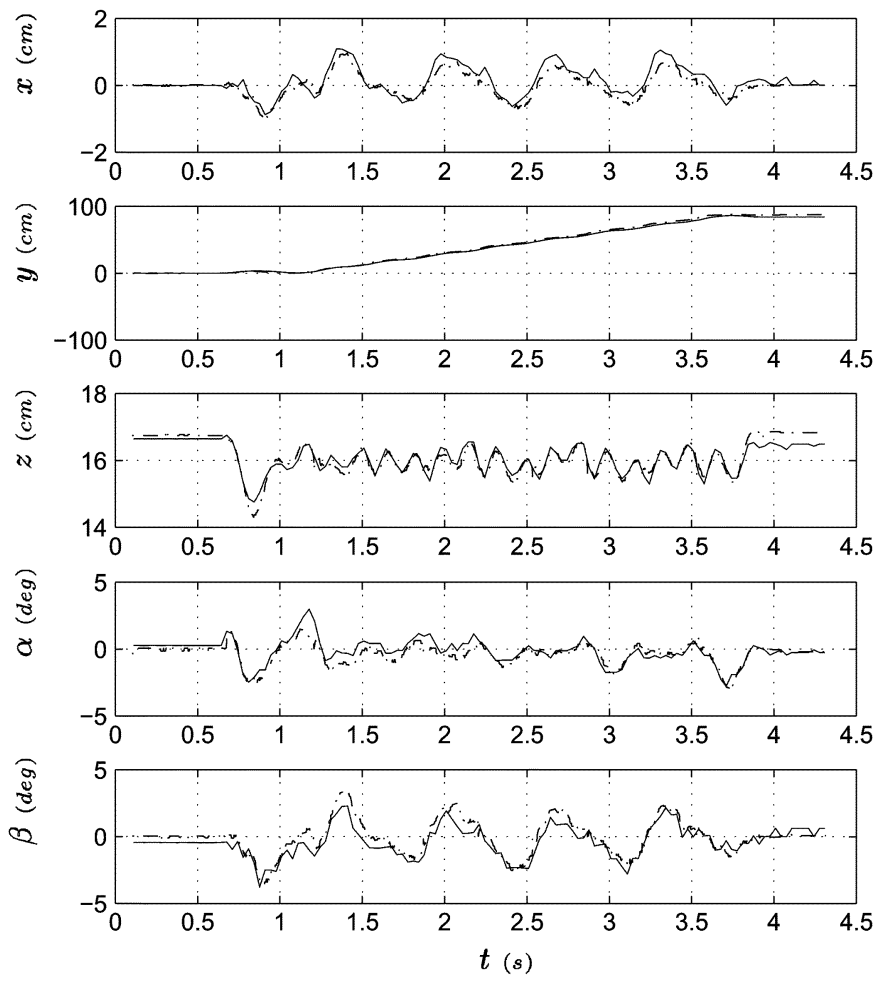

Fig. 7. Pose states measured by GTMS (solid line) and computed according to our algorithm (dashed line). Note, as explained at the end of Section II-B that the $x$ and $y$ computed (dashed) traces represent the result of the high level "Odometry via Composition" algorithm while the $z, \alpha$, and $\beta$ traces represent the result of the within stride computation described in Section II-A.

and GTMS, given by $\zeta(\mathbf{d}, \hat{\mathbf{d}}):=\sqrt{\left(\|\mathbf{d}-\hat{\mathbf{d}}\|_{2}^{2} / M\right)}$ where $\mathbf{d}=\left(\mathbf{d}_{1}, \ldots, \mathbf{d}_{M}\right)$ and $\mathbf{d}_{i}:=\left(x_{i}, y_{i}, z_{i}, \alpha_{i}, \beta_{i}\right)$ represents the configuration trajectory with length $M$ from the GTMS, while $\hat{d}$ denotes the corresponding configuration trajectory estimate output from the algorithm.

A typical run yields two sets of data for each configuration variable: GTMS measurements (solid line) and algorithm outputs (dashed line). Fig. 7 shows the comparison of these plots for each configuration variable for a typical run over multiple steps. In Table III, we compute the rms error for each configuration variable as well as the mean (avg) and standard deviation (std) of the rms error for a typical experiment set which contains 
TABLE III

RMS ERROR OF THE BODY POSE COMPUTATIONAL ALgORITHM OVER Multiple StePs (MEdium SPEED, $0.35 \mathrm{~m} / \mathrm{s}$ )

\begin{tabular}{c|c|c|c|c|c|c|c}
\hline Run \# & \multicolumn{9}{|c|}{ State } & \multicolumn{2}{c}{ Reference } \\
& $\begin{array}{c}x \\
(\mathrm{~cm})\end{array}$ & $\begin{array}{c}\boldsymbol{z} \\
(\mathrm{cm})\end{array}$ & $\begin{array}{c}\boldsymbol{z} \\
(\mathrm{cm})\end{array}$ & $\begin{array}{c}\alpha \\
(\mathrm{deg})\end{array}$ & $\begin{array}{c}\boldsymbol{\beta} \\
(\mathrm{deg})\end{array}$ & $\begin{array}{c}\boldsymbol{c} \\
(\mathrm{cm})\end{array}$ & $\boldsymbol{v}$ \\
\hline \hline Run \#1 & 0.56 & 1.52 & 0.17 & 0.50 & 0.63 & 85.1 & 8 \\
\hline Run \#2 & 0.57 & 1.98 & 0.28 & 0.64 & 0.80 & 75.0 & 7 \\
\hline Run \#3 & 0.33 & 1.20 & 0.22 & 0.78 & 0.81 & 75.2 & 7 \\
\hline Run \#4 & 0.27 & 1.46 & 0.24 & 0.54 & 0.69 & 96.8 & 9 \\
\hline Run \#5 & 0.30 & 1.94 & 0.19 & 0.53 & 0.64 & 84.2 & 8 \\
\hline avg & 0.41 & 1.42 & 0.22 & 0.60 & 0.71 & 83.3 & 7.8 \\
\hline std & 0.13 & 0.32 & 0.04 & 0.10 & 0.08 & 8.0 & 0.7 \\
\hline
\end{tabular}

TABLE IV

RMS ERROR OF THE BODY POSE COMPUTATIONAL ALGORITHM Over Multiple Steps at VARYing SPEeds Over CardBoard $\left(\mu_{s}=0.65, \mu_{k}=0.60\right)$

\begin{tabular}{|c|c|c|c|c|c|c|c|}
\hline \multirow[t]{2}{*}{ Statistics } & \multicolumn{5}{|c|}{ State } & \multicolumn{2}{|c|}{ Reference } \\
\hline & $\begin{array}{c}x \\
(\mathrm{~cm})\end{array}$ & $\begin{array}{c}y \\
(\mathrm{~cm})\end{array}$ & $\begin{array}{c}z \\
(\mathrm{~cm})\end{array}$ & $\begin{array}{c}\alpha \\
(\mathrm{deg})\end{array}$ & $\begin{array}{c}\beta \\
(\mathrm{deg})\end{array}$ & $\begin{array}{c}\nu \\
(\mathrm{cm})\end{array}$ & $v$ \\
\hline \multicolumn{8}{|c|}{ Slow walking speed $(0.25 \mathrm{~m} / \mathrm{s})$} \\
\hline avg & 0.42 & 4.53 & 0.30 & 0.78 & 0.73 & 84.0 & 8.4 \\
\hline std & 0.13 & 0.80 & 0.08 & 0.09 & 0.06 & 6.2 & 0.5 \\
\hline \multicolumn{8}{|c|}{ Medium walking speed $(0.35 \mathrm{~m} / \mathrm{s})$} \\
\hline avg & 0.41 & 1.42 & 0.22 & 0.60 & 0.71 & 83.8 & 7.8 \\
\hline std & 0.13 & 0.32 & 0.04 & 0.10 & 0.08 & 8.0 & 0.7 \\
\hline \multicolumn{8}{|c|}{ Fast walking speed $(0.51 \mathrm{~m} / \mathrm{s})$} \\
\hline avg & 0.39 & 1.41 & 0.27 & 0.63 & 0.68 & 84.8 & 7.4 \\
\hline std & 0.05 & 0.25 & 0.04 & 0.08 & 0.08 & 6.5 & 0.7 \\
\hline
\end{tabular}

five runs. ${ }^{9}$ We also include GTMS measured elapsed distance $\nu$ and number of tripod strides for each run $v$, as reference.

To evaluate the robustness of the body pose computation, we run the experiment sets in two different scenarios: 1) at widely varying speeds described in Section IV-A and 2) over dramatically different ground conditions described in Section IV-B. We also compare the performance to that of sensorless schemes-both legged as well as on a wheeled version of the robot-using GTMS measurements of elapsed distance described in Section IV-C.

\section{A. Performance at Varying Speeds}

Three sets of experiments measure the effects of walking speed on pose estimation performance: slow $(0.25 \mathrm{~m} / \mathrm{s})$, medium $(0.35 \mathrm{~m} / \mathrm{s})$, and fast $(0.51 \mathrm{~m} / \mathrm{s})$. All of these operating regimes fall into the continuous contact family that our algorithm presumes. However, the duration of the double stance and the magnitude of the ground reaction forces differ as the speed changes resulting in different levels of error in frame composition during the double-stance phase.

Table IV summarizes the results for the speed experiments. At all speeds, small rms error values compared to the robot size $(50 \mathrm{~cm} \times 25 \mathrm{~cm} \times 15 \mathrm{~cm})$ indicate successful pose computation where the mean error in angular states remain less than $1^{\circ}$ and that of lateral $(x)$ and vertical $(z)$ positions are less than $1 \mathrm{~cm}$. Since the slippage during double stance has significant impact on the fore/aft $(y)$ direction of the robot, the corresponding rms error is the largest. We observe that the error in the fore/aft position decreases with increasing speed. This is due to two changes:

\footnotetext{
${ }^{9}$ Performance of single tripod steps is detailed in Appendix VI.
}

TABLE V

RMS ERRor OF THE BODY POSE COMPUTATIONAL ALGORITHM OvER Multiple StePs OVER DifFERENT GROUND CONDITIONS AT SLOW WALKING SPEED $(0.25 \mathrm{~m} / \mathrm{s})$

\begin{tabular}{|c|c|c|c|c|c|c|c|}
\hline \multirow[t]{2}{*}{ Statistics } & \multicolumn{5}{|c|}{ State } & \multicolumn{2}{|c|}{ Reference } \\
\hline & $\begin{array}{c}\boldsymbol{x} \\
(\mathrm{cm})\end{array}$ & $\begin{array}{c}y \\
(\mathrm{~cm})\end{array}$ & $\begin{array}{c}z \\
(\mathrm{~cm})\end{array}$ & $\begin{array}{c}\alpha \\
(\mathrm{deg})\end{array}$ & $\begin{array}{c}\beta \\
(\mathrm{deg})\end{array}$ & $\begin{array}{c}\nu \\
(\mathrm{cm})\end{array}$ & $v$ \\
\hline \multicolumn{8}{|c|}{ Cardboard $\left(\mu_{s}=0.65, \mu_{k}=0.60\right)$} \\
\hline avg & 0.42 & 4.53 & 0.30 & 0.78 & 0.73 & 84.0 & 8.4 \\
\hline std & 0.13 & 0.80 & 0.08 & 0.09 & 0.06 & 6.2 & 0.5 \\
\hline \multicolumn{8}{|c|}{ Plastic $\left(\mu_{s}=0.33, \mu_{k}=0.27\right)$} \\
\hline avg & 0.40 & 4.45 & 0.51 & 0.83 & 0.65 & 79.0 & 8.0 \\
\hline std & 0.08 & 0.82 & 0.05 & 0.05 & 0.06 & 6.2 & 0.6 \\
\hline \multicolumn{8}{|c|}{ Plastic covered with wet soap $\left(\mu_{s}=0.20, \mu_{k}=0.11\right)$} \\
\hline avg & 1.39 & 9.72 & 0.45 & 0.63 & 1.12 & 73.8 & 7.4 \\
\hline std & 0.22 & 1.30 & 0.07 & 0.07 & 0.22 & 5.6 & 0.5 \\
\hline \multicolumn{8}{|c|}{ Plastic covered with dry soap $\left(\mu_{s}=0.07, \mu_{k}=0.05\right)$} \\
\hline avg & 1.82 & 9.40 & 0.42 & 0.63 & 0.65 & 78.6 & 8.2 \\
\hline std & 0.28 & 0.56 & 0.07 & 0.09 & 0.05 & 3.1 & 0.4 \\
\hline
\end{tabular}

1) an increase in speed causes a shorter double-stance period improving the accuracy of the sequential composition computation and 2) ground reaction force magnitude increases with speed, decreasing the likelihood of slippage during stance.

\section{B. Performance Over Different Ground Conditions}

In a second set of experiments, we investigate the effects of of varying surface "stickiness" that we expect to impact most significantly the accuracy of our pose estimation algorithm. For this study, we appeal to the standard macroscopic model of "stickiness" [30] and characterize surfaces according to their static (stiction) $\mu_{s}$ and kinetic (sliding) $\mu_{k}$ friction coefficients.

We empirically determine the friction coefficient of surfaces to be used in these experiments by placing the robot on the surface of interest in the standing pose and pulling in the fore/aft direction. The exerted force is measured by a 1-DOF force sensor (Cooper Instruments, LFS260), and the collected data are analyzed to determine the static $\mu_{s}$ and kinetic $\mu_{k}$ friction coefficients.

In these experiments, the robot walks at slow speed $(0.25 \mathrm{~m} / \mathrm{s})$ over four types of surfaces: cardboard $\left(\mu_{s}=0.65, \mu_{k}=0.60\right)$, plastic $\left(\mu_{s}=0.33, \mu_{k}=0.27\right)$, plastic covered with wet soap $\left(\mu_{s}=0.20, \mu_{k}=0.11\right)$, and plastic covered with dry soap $\left(\mu_{s}=0.07, \mu_{k}=0.05\right)$. Table $\mathrm{V}$ summarizes the results of this experiment set. As the friction coefficient decreases, we observe significant deterioration in the accuracy of the horizontal components $(x, y)$ of the COM. This is a direct result of the increase in slippage. The vertical component $(z)$ of the COM and angular configuration variables $(\alpha, \beta)$ are not affected as severely since these are invariant with respect to COM horizontal translation (the most significant component of a "slipping" robot's motion), and, moreover, their computation only depends on the current single-stance phase measurements but not on any of the previous ones. In general, except on the most adversarial slippery surfaces such as the soaped plastic we specially prepared for this study, the algorithm performs well and consistently over normal ground conditions: for example, those characterizing cardboard or plastic that seem to typify the static friction coefficients we observe $\left(0.3 \leq \mu_{s} \leq 0.7\right)$ on most of the indoor terrain that the robot's rubber toes encounter. 
TABLE VI

ODOMETRY PERFORMANCE AT VARYING SPEEDS AND OVER DIFFERENT Ground Conditions (RHex With Leg Strain Based Pose SEnsor Versus SENSORLESS RHEX VERSUS WHEELED RHEX)

\begin{tabular}{|c|c|c|c|c|c|c|c|}
\hline \multirow[t]{3}{*}{ Statistics } & \multicolumn{5}{|c|}{ Legged RHex } & \multicolumn{2}{|c|}{ Wheeled RHex } \\
\hline & \multirow{2}{*}{$\begin{array}{c}\text { GTMS } \\
\nu \\
(\mathrm{cm})\end{array}$} & \multicolumn{2}{|c|}{ sensorless } & \multicolumn{2}{|c|}{ pose-sensor-based } & \multirow{2}{*}{$\begin{array}{c}\text { GTMS } \\
\nu_{w} \\
(\mathrm{~cm}) \\
\end{array}$} & \multirow[b]{2}{*}{$\begin{array}{l}\kappa_{w} \\
(\%)\end{array}$} \\
\hline & & 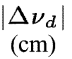 & $\begin{array}{c}\kappa_{d} \\
(\%)\end{array}$ & $\begin{array}{c}\left|\Delta \nu_{l}\right| \\
(\mathrm{cm})\end{array}$ & $\begin{array}{c}\kappa_{l} \\
(\%)\end{array}$ & & \\
\hline \multicolumn{8}{|c|}{ Slow walking speed $(0.25 \mathrm{~m} / \mathrm{s})$ on cardboard $\left(\mu_{s}=0.65, \mu_{k}=0.60\right)$} \\
\hline avg & 84.0 & 17.9 & 21.4 & 5.1 & 6.1 & 87.6 & 0.3 \\
\hline std & 6.2 & 0.7 & 2.0 & 1.9 & 0.7 & 3.3 & 0.09 \\
\hline \multicolumn{8}{|c|}{ Medium walking speed $(0.35 \mathrm{~m} / \mathrm{s})$ on cardboard $\left(\mu_{s}=0.65, \mu_{k}=0.60\right)$} \\
\hline avg & 83.8 & 17.6 & 21.1 & 1.8 & 2.2 & 83.3 & 0.3 \\
\hline std & 8.0 & 1.8 & 1.0 & 0.8 & 1.0 & 1.8 & $0.07)$ \\
\hline \multicolumn{8}{|c|}{ Fast walking speed $(0.51 \mathrm{~m} / \mathrm{s})$ on cardboard $\left(\mu_{s}=0.65, \mu_{k}=0.60\right)$} \\
\hline avg & 84.8 & 13.9 & 16.3 & 1.5 & 1.7 & 80.8 & 0.5 \\
\hline std & 6.5 & 3.1 & 2.9 & 0.6 & 0.6 & 0.9 & $0.26)$ \\
\hline \multicolumn{8}{|c|}{ Slow walking speed on plastic $\left(\mu_{s}=0.33, \mu_{k}=0.27\right)$} \\
\hline avg & 79.0 & 18.0 & 22.8 & 5.3 & 6.6 & 87.6 & 0.3 \\
\hline std & 6.2 & 1.9 & 1.8 & 3.4 & 4.4 & 5.2 & 0.08 \\
\hline \multicolumn{8}{|c|}{ Slow walking speed on plastic covered with wet soap $\left(\mu_{s}=0.20, \mu_{k}=0.11\right)$} \\
\hline avg & 73.8 & 16.0 & 21.8 & 6.0 & 8.2 & 90.2 & 1.7 \\
\hline std & 5.6 & 0.6 & 1.5 & 0.4 & 0.7 & 4.0 & 0.22 \\
\hline \multicolumn{8}{|c|}{ Slow walking speed on plastic covered with dry soap $\left(\mu_{s}=0.07, \mu_{k}=0.05\right)$} \\
\hline avg & 78.6 & 20.9 & 26.5 & 14.0 & 17.8 & 93.2 & 1.2 \\
\hline std & 3.1 & 2.5 & 2.9 & 0.7 & 0.6 & 5.1 & 0.43 \\
\hline
\end{tabular}

\section{Odometry Performance}

Although online estimation of within-stride state represents our chief motivation for developing this body pose sensor, we have noted in the Introduction a large amount of literature in the field of mobile robotics concerned with odometry and observed in Section II that the assumptions of the walking gait over level terrain allow the computation of legged odometry using the procedure that we described therein.

Table VI compares our leg strain-based odometry estimates (by reference to discrepancies with GTMS measurements of elapsed distance) with sensorless schemes for the legged machine as well as on a wheeled implementation of the Robot pictured in Fig. 3(a). With no sensing apart from motor shaft measurements, blind odometry estimates result from counting the number of leg cycles and multiplying by a previously calibrated distance-per-cycle constant. Of course, this is the traditional approach to odometry in wheeled vehicles as well. We ran calibration tests for RHex and a wheeled implementation of the Robot, counting the number of motor shaft cycles over the same long flat surface to get the best possible conversion constant. The table presents discrepancies $\kappa(\%)(=|\Delta \nu| / \nu)$ as a percentage of the GTMS measured elapsed distance $\nu$ for each of the three odometry methods: sensorless legged, pose-based legged, and sensorless wheeled. The results show that the leg strain-based odometry from body pose measurements is greatly superior to the blind predictions of the open-loop scheme by nearly an order of magnitude at the higher speeds where the inaccuracies of double support have less effect. The dynamical nature of legged walking (even in the absence of an aerial phase, RHex's gaits exhibit a significant interchange of body kinetic and leg spring potential energy in stance) causes speed variations during locomotion that incur significantly more slippage (exacerbated at slower gaits by prolonged double support) than the far smoother ride afforded by wheels. Thus, our sensorbased legged odometry is significantly less accurate than the blind results of counting motor shaft revolutions on the wheeled version of the same machine.

\section{CONCLUSION}

We have introduced a full-body pose estimator for a walking hexapod robot based on the kinematic configuration of its legs structured in a two-layer hierarchy. The lower layer relates leg configuration to body pose within a single stance while the higher layer recursively composes consecutive single-stance measurements to achieve complete legged odometry-continuous pose computation across multiple steps. We have implemented this algorithm on the robot RHEX [2] utilizing strain measurements of its four-bar legs [27] communicated over a wireless communications network [29].10

Using a high-speed visual GTMS, we have shown that the leg configuration model used to interpret the strain data achieves very high accuracy in realistic operating conditions (e.g., effective length errors of less than $2 \%$ ). Using a separate (conventional frame rate) visual GTMS, we have evaluated the performance of the resulting low-level single-stance pose estimator and the high-level legged odometry system at various speeds and conditions of surface friction. The body pose estimator is shown to perform well at all speeds over normal ground conditions-achieving, for example, five times more accurate legged odometry than computed from averaged open-loop distanceper-stride estimates. The estimator continues to function well over a variety of ground conditions, with the onset of significant performance degradation on the most slippery surfaces (soaped plastic) whose coefficient of friction is less than a third that of normal linoleum.

In its present form, the pose computation algorithm cannot function if the operating regime includes an aerial phase as is typical of RHex's most useful dynamical gaits [9]. To remedy this shortcoming, our future work will introduce other sensor modalities such as linear accelerometers and rotational rate gyroscopes to complement the leg kinematic configuration sensor introduced here. This multiple array of sensor modalities will not only allow us to perform pose estimation during aerial phases but also enable us to detect and correct errors resulting from slippage, the primary source of inaccuracy in the present sensor.

\section{APPENDIX I \\ PLANARITY FUNCTION}

Planarity function is a function we use to determine the "planarity" of a set of points by finding the least squared error to a specific plane which is parameterized with minimum least squared error to these points.

Given a set of $N$ points in the three-dimensional (3-D) space $\mathrm{s}_{i}, i=1, \ldots, N$, by defining the covariant matrix as

$$
\mathbf{P}:=\sum_{i=1}^{N} \mathbf{s}_{i}^{T} \mathbf{s}_{i}-\overline{\mathbf{s}}^{T} \overline{\mathbf{s}} \quad \text { where } \overline{\mathbf{s}}:=\frac{1}{N} \sum_{i=1}^{N} \mathbf{s}_{i}
$$

then the eigenvector $\mathbf{v}$, associated with the smallest eigenvalue in $\mathbf{P}$ by function $\mathbf{V}: \mathbf{v}:=\mathbf{V}(\mathbf{P})$, represents the normal direction of the plane with minimum least squared error to these points.

\footnotetext{
${ }^{10}$ Note, in consequence of RHex's constrained kinematics, as explained in Section IV, we have not exercised its yaw degree of freedom in this study.
} 


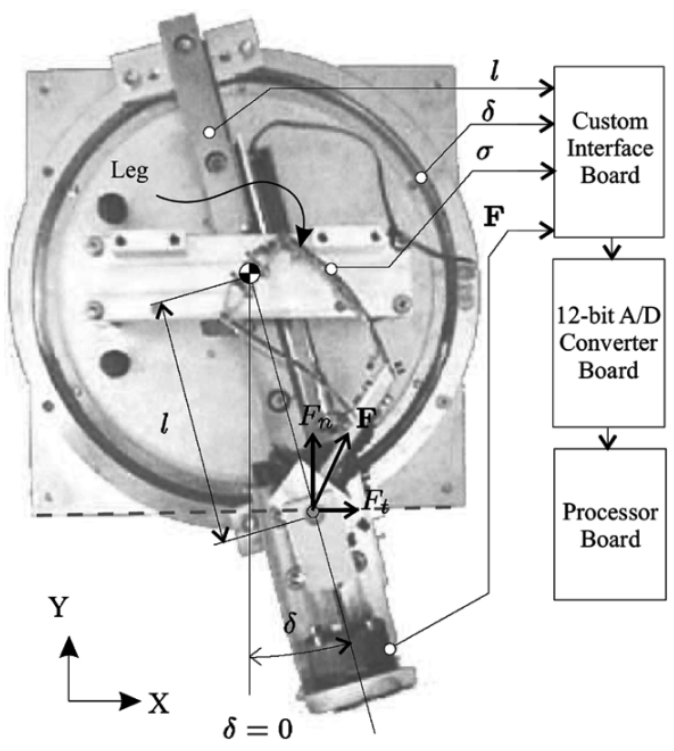

Fig. 8. Benchtop data collection setup testing a four-bar leg. During an experiment, we measure the position of the toe in the clamp coordinate system $\left[\begin{array}{ll}l & \delta\end{array}\right]^{T} \in \mathcal{C}$, and the two components of the ground reaction force $\mathbf{F}:=\left[\begin{array}{ll}F_{n} & F_{t}\end{array}\right]^{T}$, as indicated on the picture. A block diagram of the PC/104 stack performing the data collection is illustrated on the right.

With the knowledge that the plane passes the mean of the points, we can define the planarity function as

$$
\rho(\mathbf{s}):=\frac{1}{N} \sum_{i=1}^{N}\left[\left(\mathbf{s}_{i}-\overline{\mathbf{s}}\right) \cdot \mathbf{V}(\mathbf{P})\right]^{2}
$$

which yields the mean squared distance of the points around the best common plane. ${ }^{11}$

In our application, we empirically set a threshold $\bar{\rho}$ on this planarity function $\rho(\mathbf{s})$, with six toe coordinates in body coordinate system $\mathbf{s}_{i}, i=1 \ldots 6$ as the inputs to determine the double-stance phase in order to compute the transformations between tripod coordinate systems of consecutive single-stance phases.

\section{APPENDIX II \\ Benchtop Data Collection Setup}

A benchtop setup [28], illustrated in Fig. 8, facilitates the data collection for the kinematic leg model identification studies in Section III-C. This section describes the design and operation of this device.

In this 2-DOF setup, the hip clamp of the tested four-bar leg is rigidly mounted at the origin of the setup fixing both its position and orientation satisfying: 1 ) the leg plane $\mathcal{L}$ coincides with the table plane and 2) the toe vector at rest, $\delta=0$, points vertically downward. The virtual ground is a horizontal line passing through the toe which is clamped to a revolute joint. The toe clamp is mounted on a turn table centered at the origin where a linear slider aligned with the toe vector. Position of the toe, $\mathbf{u}=\left[\begin{array}{ll}l & \delta\end{array}\right]^{T}$, in the polar clamp coordinate system $\mathcal{C}$ is measured by linear (Midori LP-50F) and rotary (Midori CPP-35B)

\footnotetext{
${ }^{11}$ In general, planarity can also be determined by the relation between eigenvalues in covariant matrix $\mathbf{P}$, but we prefer to use (6) because it also delivers physical measure.
}

potentiometers along these two sliders. A three-axis force sensor (Bokam DX-480) at the toe clamp measures the ground reaction force $\mathbf{F}=\left[\begin{array}{ll}F_{t} & F_{n}\end{array}\right]^{T}$ at the toe in the world coordinate system $\mathcal{W}$. The strain $\sigma$ measured by the strain gauge installed on the back compliant member is interfaced by an instrumental amplifier in the custom interface board in the same manner as it is done in LegNet leg modules in realistic operating conditions.

Acquisition of the table data is performed by a PC104 stack containing: 1) Lippert Automationstechnik S-104PCRR2-VEPS300 PC104 $300 \mathrm{MHz}$ CPU board with 256-MB RAM and 512-MB compact flash running QNX6.0 real-time OS; 2) Micro/Sys MPC550 12-bit analog-to-digital converter board; 3) Real Time Device EPWR104-HR25/25 W PC104 $5 \mathrm{~V} / 12 \mathrm{~V}$ power supply; 4) VersaLogic PCM-3115 PC104 PCMCIA adapter with Avaya 128RC4 wireless ethernet; and 5) a custom-built interface board between sensors and the MPC550 I/O board.

Sensors are sampled at $1 \mathrm{kHz}$ while the position of the toe is manually manipulated at slow speeds. Sensor data, which are composed of the toe position in the clamp coordinate system $\mathbf{u} \in \mathcal{C}$ and the ground reaction force $\mathbf{F}$, at the toe in the world coordinate system are filtered by a low-pass filter with cut-off frequency at $20 \mathrm{~Hz}$ to eliminate high-frequency sensor noise. Resulting data are stored for off-line model parameter identification.

\section{APPENDIX III Benchtop Data Cleaning Procedure}

Benchtop data cleaning procedure is applied in order to obtain uniform distributed data from raw nonuniform data points as well as to extract a physical relevant subset of data points suitable for robot normal walking.

The first step in data cleaning will be to obtain a data set that is uniformly distributed in leg plane $\mathcal{L}$ to eliminate spurious weighting effect of the uneven distribution of the raw data in fitting computations. For this purpose, we employ an averaging procedure. In this method, we partition the leg plane $\mathcal{L}$ into a uniform grid of rectangular cells. For those cells that contain nonzero raw data points, we place a single data point at the center of the cell whose ground reaction force and strain are given by the average of the corresponding values of the raw points contained in that cell. Fig. 9(top right) shows the result of this procedure applied to the raw data in Fig. 9(top left).

The second step in the data cleaning process is to choose the physically relevant subset of the database that correspond to the ground contact situation suitable for robot walking case. Our object of interest, which is characterized by two force constraints: 1) a support condition requiring that the normal ground reaction force $F_{n}$ be in the upward direction supporting the weight of the body, $F_{n}>0$ and 2) friction condition requiring that the magnitude of the friction force $F_{t}$ be smaller than that of the maximum static friction force $F_{s}, F_{t}<F_{s}$. We filter the uniform data in Fig. 9 (top right) in two consecutive steps where Fig. 9 (bottom left) is the result of the support condition filter and Fig. 9 (bottom right) is final physically relevant data set after application of the friction constrain filter. We notice that the locus of the physically relevant points lie in a very thin set in the leg plane $\mathcal{L}$, 

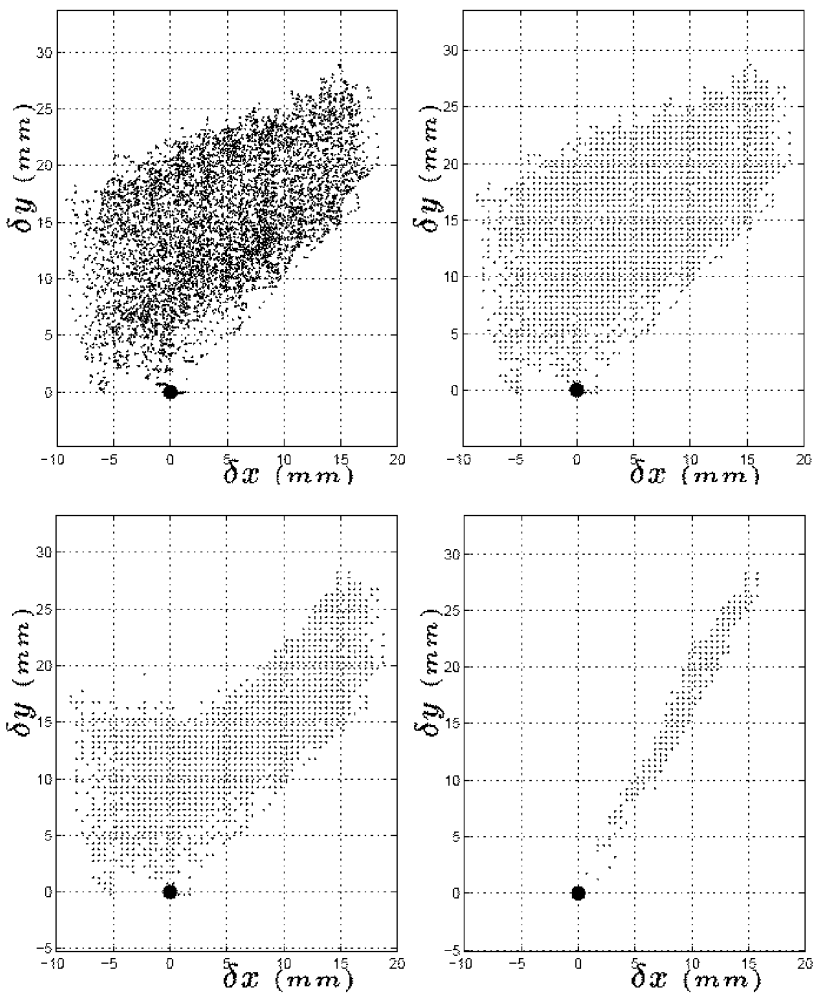

Fig. 9. Scatter-plots of toe positions: (top left) raw data; (top right) uniformly distributed data; (bottom left) data with suitable normal ground reaction force satisfying the support condition; and (bottom right) final physical relevant points with suitable relation between friction force and normal force satisfying the friction condition.

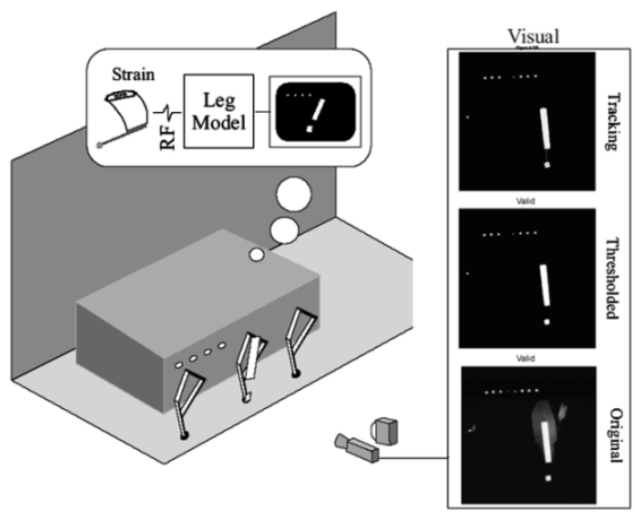

Fig. 10. High-speed sagittal plane ground truth experimental test station.

which acts as the basis of our 1-DOF leg model assumption. The maximum static frictional force is given by $F_{s}=\mu_{s} F_{n}$ where $\mu_{s}$ is the coefficient of static friction which is empirically measured to be 0.65 for the four-bar legs over cardboard surface.

\section{APPENDIX IV \\ High-SpeEd MEASUREMENT OF LEG CONFIGURATION}

This section describes a setup for high-speed ground truth measurements of kinematic configuration of a leg, represented by the position of its toe, during realistic operating conditions.

Fig. 10 illustrates the experimental setup where robot walks along a $2 \mathrm{~m} \times 1 \mathrm{~m}$ runway. A high-speed black-and-white analog camera (RadLake HR-1000) is located $1 \mathrm{~m}$ away from the path viewing the robot in the sagittal plane at $125 \mathrm{fps}$. In order to simplify the off-line analysis of the visual data, the runway and its background as well as the frame of the robot are all covered with black cardboard. We install two reflective markers (3 M) on the leg: 1) a toe marker, $10 \mathrm{~mm} \times 10 \mathrm{~mm}$ square reflective piece at the toe and 2) a ruler marker, 100 $\mathrm{mm} \times 10 \mathrm{~mm}$ rectangular piece extending from the hip attachment point toward the position of the toe at rest. A low-power halogen lamp is located near the camera to illuminate the scene. LEDs are installed on the robot within camera view for data synchronization.

An experiment starts with the robot placed at the beginning of the runway in standing pose ready to walk. Visual recording is initiated first followed by the activation of the logger on the robot. As soon as the LEDs on the robot indicate the start of the logging of strain, the user commands the robot to walk until it gets out of the camera view.

Recorded video is converted into a sequence of frames. Starting from the frame where the LEDs are on for the first time, the frame sequence is feed to a MATLAB script. Each gray-scale frame is converted to black and white by setting threshold and the objects in the image are distinguished from each other by connected components method. The two markers, the toe and the ruler, are identified based on their geometric properties. The effective leg length $l$ is the distance from the top of the ruler marker to the center of the toe marker, and the deflection angle $\delta$ is the angle between the major axis of the ruler marker and the line connecting the top of the ruler to the center of the toe. Note that both the resulting visual measurements and the strain measurements recorded on the robot start at the same instant since they are synchronized by the activation of LED.

\section{APPENDIX V \\ DOF GROUND TRUTH MEASUREMENT SETUP}

To evaluate the performance of the body pose computation algorithm in realistic operating conditions we utilize a GTMS [31] - a 3-D visual tracking system for multiple markers.

Fig. 11 illustrates the key components of the GTMS setup. Two analog cameras (SONY XC-77) are located on both sides of a $3 \mathrm{~m} \times 3 \mathrm{~m}$ runway looking down from a height of $2 \mathrm{~m}$ combined with two halogen lamps installed next to each camera for illumination. The video streams are synchronized by two junction boxes (SONY, JB-77), digitized by two frame-grabber cards (Data Translation DT3155) at $30 \mathrm{~Hz}$, and imported into a Pentium-based PC running Linux as our computational resource. To ease the detection of markers, the runway and the robot are covered with black cardboard. Three markers made by spherical balls covered with reflective tape $(3 \mathrm{M})$ are installed on top of the robot. For synchronization between robot actions with the visual recording, an LED referred to as the sync-LED installed on top of the robot acts as a controllable fourth marker which is visible to the cameras as well.

An experiment starts with the robot in a standing pose in the beginning of the runway within the field of view of both cameras. The sync-LED, off in the beginning, is turned on, indicating the $t=0$, for the run right after visual recording 


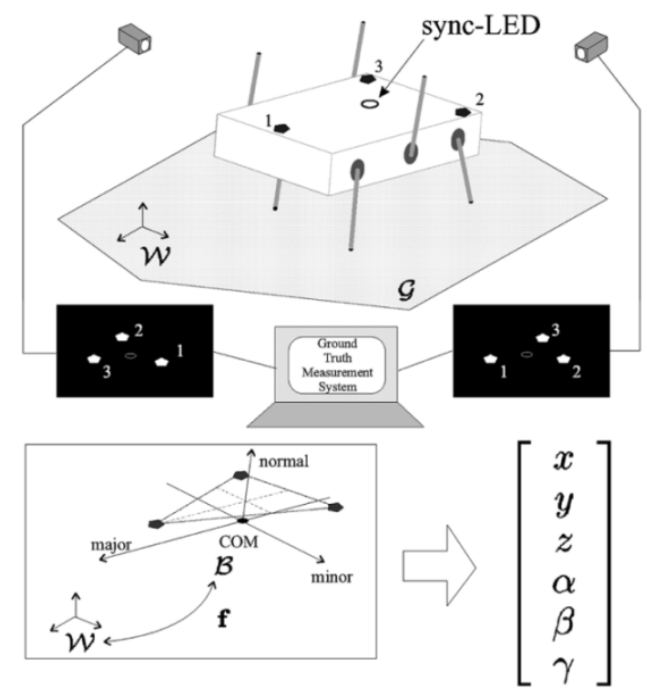

Fig. 11. Two cameras track the three markers on the body which are depicted by numbered pentagons. We use a fourth marker (an LED) on the robot, shown as an empty circle, to synchronize the logging on the robot with the visual data. An off-line system computes body pose with respect to the world coordinate system $\mathcal{W}$.

TABLE VII

AbSolute MaXimum ERror In GTMS MEASUREMENT OF Body Pose

\begin{tabular}{c||c|c|c|c|c|c}
\hline state & $x$ & $y$ & $z$ & $\alpha$ & $\beta$ & $\gamma$ \\
\hline unit & $\mathrm{cm}$ & $\mathrm{cm}$ & $\mathrm{cm}$ & $\mathrm{deg}$ & $\mathrm{deg}$ & $\mathrm{deg}$ \\
\hline abs max & 0.5 & 0.5 & 0.5 & 1.71 & 2.79 & 1.30 \\
\hline
\end{tabular}

starts. For the following $9 \mathrm{~s}$, the maximum length of the visual recording limited by the PC's buffer, user commands the robot to walk in a straight line while GTMS records digital video streams from two cameras into PC's memory. After the recording is complete, the raw visual data are processed by an off-line tracking program which triangulates the positions of all the markers starting from the frame when the sync-LED is first detected, and the final markers' trajectories represented in the world coordinate system, $\mathcal{W}$, are saved in a file for follow-up computations to transform into robot pose based on known geometry relation between markers and COM.

Triangulation performance of GTMS is measured at a set of points scattered within the field of view of the cameras. We compare the output of the triangulation to the known physical location for each marker and determine the absolute maximum error in triangulation to be $5 \mathrm{~mm}$. In the above procedure, error in the vertex positions leads to error in body pose states. For each state, we construct the worst case scenario that yields the largest error given a triangulation error. Plugging in the empirically measured triangulation error, we obtain the absolute maximum error for each state as listed in Table VII.

\section{APPENDIX VI \\ Single-Stance Phase Pose Computation}

In the performance analysis of the pose computation, we choose to follow the hierarchical structure of the algorithm. This section will focus on the error in the low-level individual single-stance phases without the complications introduced by the sequential composition in the higher level.
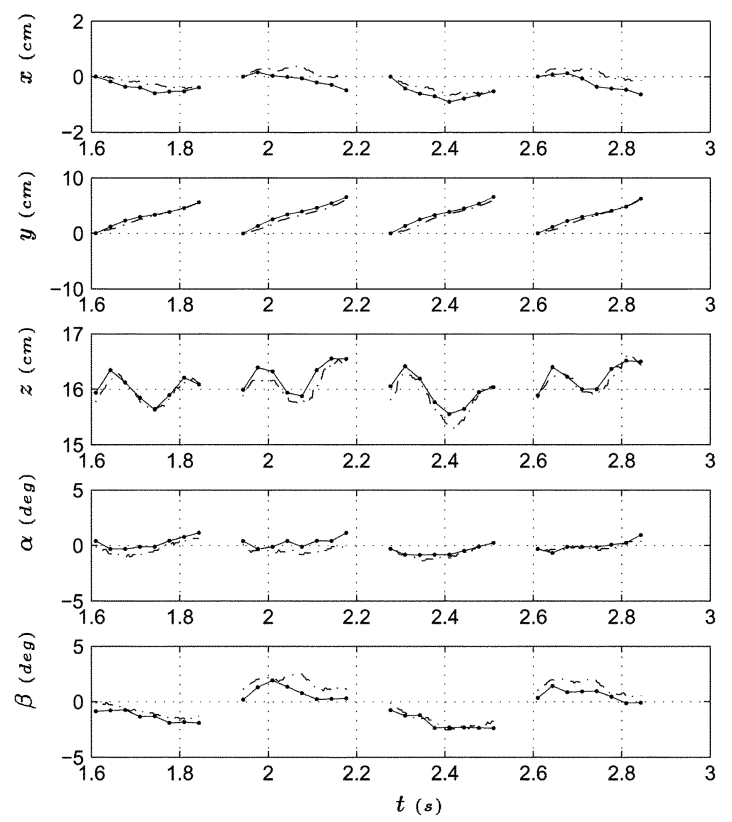

Fig. 12. Pose states measured by GTMS (solid line) and computed according to our algorithm (dashed line) for single-stance body pose computation.

TABLE VIII

RMS ERRor of Single-STANCE Body PoSe COMPUTATION IN A TYPICAL RUN (MEDIUM SPEED, $0.35 \mathrm{~m} / \mathrm{s}$ )

\begin{tabular}{c|c|c|c|c|c}
\hline Run \# & & \multicolumn{4}{c}{ State } \\
& $\begin{array}{c}x \\
(\mathrm{~cm})\end{array}$ & $\begin{array}{c}\boldsymbol{y} \\
(\mathrm{cm})\end{array}$ & $\begin{array}{c}\alpha \\
(\mathrm{cm})\end{array}$ & $\begin{array}{c}\beta \\
(\mathrm{deg})\end{array}$ & $(\mathrm{deg})$ \\
\hline \hline Run \#1 & 0.16 & 0.40 & 0.13 & 0.44 & 0.58 \\
\hline Run \#2 & 0.22 & 0.35 & 0.19 & 0.56 & 0.89 \\
\hline Run \#3 & 0.27 & 0.33 & 0.19 & 0.54 & 0.77 \\
\hline Run \#4 & 0.24 & 0.44 & 0.16 & 0.42 & 0.78 \\
\hline Run \#5 & 0.27 & 0.52 & 0.13 & 0.50 & 0.70 \\
\hline avg & 0.23 & 0.41 & 0.16 & 0.49 & 0.74 \\
\hline std & 0.04 & 0.07 & 0.03 & 0.05 & 0.10 \\
\hline
\end{tabular}

These experiments are executed similarly to those in Section IV, however, the analysis of the data differs slightly. We are only concerned with the data during single-stance phases and will be ignoring the double-stance intervals. We set the origin of both the world coordinate system $\mathcal{W}$ as well as the tripod coordinate system $\mathcal{T}$, according to the COM of the robot measured by the GTMS in the beginning of each single-stance phase. Based on this common reference, the output of the algorithm and the measurements of the GTMS are plotted against each other. Fig. 12 shows such a set of plots for a typical run. For each single-stance phase, we evaluate the rms error for each configuration variable. Table VIII summarizes the rms error values for the data illustrated in Fig. 12. The mean and standard deviation of rms error for each state is also computed.

\section{ACKNOWLEDGMENT}

The authors would like to thank Prof. R. B. Gillespie, Prof. A. D. Kuo, and Dr. J. Borenstein from the University of Michigan and Prof. M. R. Cutkosky from Stanford University for their valuable advice. The authors also would like to thank Dr. R. E. Groff for his advice on modeling and formulation of the planarity measure and Dr. G. C. Sharp for his visual tracking system implementation. 


\section{REFERENCES}

[1] M. Buehler, U. Saranli, and D. E. Koditschek, "Single actuator per leg robotic hexapod," U.S. Patent 6481 513, Nov. 19, 2002.

[2] U. Saranli, M. Buehler, and D. E. Koditschek, "Rhex-A simple and highly mobile hexapod robot," Int. J. Robot. Res., vol. 20, no. 7, pp. 616-631, 2001

[3] J. D. Weingarten, R. E. Groff, and D. E. Koditschek, "Automated gait generation and optimization for legged robots," in Proc. IEEE Int. Conf. Robotics and Automation, vol. 3, 2004, pp. 2153-2158.

[4] E. Z. Moore and M. Buehler, "Stable stair climbing in a simple hexapod," in Proc. 4th Int. Conf. Climbing and Walking Robos, 2001, pp. 603-610.

[5] E. Z. Moore, D. Campbell, F. Grimminger, and M. Buehler, "Reliable stair climbing in the simple hexapod 'rhex"," in Proc. IEEE Int. Conf. Robot. Automat., vol. 3, 2002, pp. 2222-2227.

[6] D. Campbell and M. Buehler, "Stair descent in the simple hexapod 'RHex',' in Proc. IEEE Int. Conf. Robot. Automat., vol. 1, 2003, pp. 1380-1385.

[7] H. Komsuoglu, D. McMordie, U. Saranli, N. Moore, M. Buehler, and D. E. Koditschek, "Proprioception based behavioral advances in a hexapod robot," in Proc. IEEE Int. Conf. Robot. Automat., vol. 4, Seoul, Korea, 2001, pp. 3650-3655.

[8] U. Saranli and D. E. Koditschek, "Back flips with a hexapodal robot," in Proc. IEEE Int. Conf. Robot. Automat., vol. 3, 2002, pp. 2209-2215.

[9] U. Saranli, "Dynamic locomotion in a hexapod robot," Ph.D. dissertation, Univ. of Michigan, Ann Arbor, Aug. 2002.

[10] P. Lin, H. Komsuoglu, and D. E. Koditschek, "A leg configuration sensory system for dynamical body state estimates in a hexapod robot," in Proc. IEEE Int. Conf. Robot. Automat., vol. 1, 2003, pp. 1391-1396.

[11] P. Lin, H. Komsuoglu, and D. E. Koditschek, "Legged odometry from body pose in a hexapod robot," in Proc. IFRR Int. Symp. Experimental Robot., to be published.

[12] M. C. Kim and W. K. Chung, "Posture estimation of a car-like mobile robot using disturbance conditions," Adv. Robot., vol. 13, no. 2, pp. 189-202, 1999 .

[13] H. Chung, L. Ojeda, and J. Borenstein, "Accurate mobile robot dead-rockoning with a precision-calibrated fiber-optic gyroscope," IEEE Trans. Robot. Automat., vol. 17, no. 1, pp. 80-84, Feb. 2001.

[14] Y. Ma, J. Kosecka, and S. S. Sastry, "Vision guided navigation for a nonholonomic mobile robot," IEEE Trans. Robot. Automat., vol. 15, no. 3, pp. 521-536, Jun. 1999.

[15] L. Moreno, J. M. Armingol, S. Garrido, A. D. L. Escalera, and M. A. Salichs, "A genetic algorithm for mobile robot localization using ultrasonic sensors," J. Intell. Robot. Syst., vol. 34, no. 2, pp. 135-154, 2002.

[16] P. Goel, S. I. Roumeliotis, and G. S. Sukhatme, "Robust localization using relative and absolute position estimate," in Proc. IEEE/RSJ Int. Conf. Intell. Robots Syst., vol. 2, 1999, pp. 1134-1140.

[17] P. Gorce, "Dynamic postural control method for biped in unknown environment," IEEE Trans. Robot. Automat., vol. 29, no. 6, pp. 616-626, Dec. 1999.

[18] H. Yoshida, K. Inoue, and Y. Mae, "Mobile manipulation of humanoid robots-Optimal posture for generating large force based on statics," in Proc. IEEE Int. Conf. Robot. Automat., vol. 3, 2002, pp. 2271-2276.

[19] H. Rehbinder and X. Hu, "Nonlinear pitch and roll estimation for walking robots," in Proc. IEEE Int. Conf. Robot. Automat., vol. 3, 2000 , pp. 2617-2622.

[20] E. Martinez, "Qualitative vision for the guidance of legged robots in unstructured environments," Pattern Recognit., vol. 34, no. 8, pp. 1585-1599, Aug. 2001.

[21] G. A. Lopes and D. E. Koditschek, "Visual registration and navigation using planar features," in Proc. IEEE Int. Conf. Robot. Automat., vol. 3, 2003, pp. 3935-3940.

[22] K. Hosoda, M. Kamado, and M. Asada, "Vision-based servoing control for legged robots," in Proc. IEEE Int. Conf. Robot. Automat., vol. 4, 1997, pp. 3154-3159.

[23] D. M. Lane, J. B. C. Davies, G. Robinson, D. J. O’Brien, J. Sneddon, E. Seaton, and A. Elfstrom, "Amadeus dextrous subsea hand: Design, modeling, and sensor processing," IEEE J. Ocean. Eng., vol. 24, no. 1, pp. 96-111, Jan. 1999.
[24] A. Inmann and M. Haugland, "An instrumented object for evaluation of lateral hand grasp during functional tasks," J. Med. Eng. Technol., vol. 25, no. 5, 2001.

[25] Y. Yu, T. Ishitsuka, and S. Tsujio, "Torque sensing of finger joint using strain-deformation expansion mechanism," in Proc. IEEE Int. Conf. Robot. Automat., vol. 2, 2003, pp. 1850-1856.

[26] R. P. Paul, Robot Manipulators-Mathematics, Programming, and Control. Cambridge, MA: MIT Press, 1983.

[27] E. Z. Moore, "Leg design and stair climbing control for the Rhex robotic hexapod,' M.S. thesis, McGill Univ., Montreal, QC, Canada, Nov. 2001.

[28] P. Lin, "Benchtop setting for compliant leg test and modeling," Univ. of Michigan, Tech. Rep., 2005. in preparation.

[29] H. Komsuoglu, "Legnet-A distributed wireless network," Univ. of Michigan, Tech. Rep., 2005. in preparation.

[30] D. Halliday and R. Resnick, Fundamentals of Physics. New York: Wiley, 1988, pp. 104-109.

[31] G. C. Sharp. (2003) Ground Truth Measurement System [Online]. Available: http://sourceforge.net/projects/gtms/

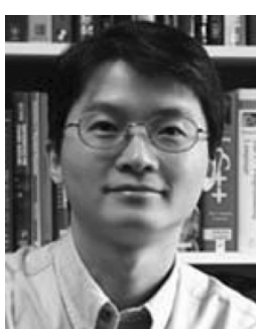

Pei-Chun Lin (S'02) received the B.S. and M.S. degrees in mechanical engineering from National Taiwan University (NTU), Taipei, Taiwan, R.O.C., in 1996 and 1998, respectively and is currently working toward the Ph.D. degree in mechanical engineering from The University of Michigan, Ann Arbor

His research interests include mechanical design, sensor design, sensor fusion, and analysis of robot dynamic behavior.

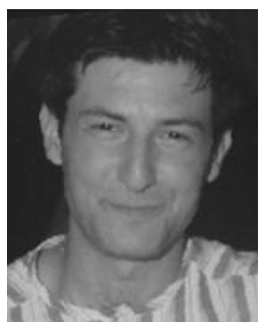

Haldun Komsuoḡlu (S'95-M'04) received the B.S degree from the Middle East Technical University (METU), Ankara, Turkey, in 1997 and the M.S. and Ph.D. degrees from The University of Michigan, Ann Arbor, in 1998 and 2004, respectively. His dissertation was entitled "Toward a formal framework for open-loop control of rhythmic tasks."

His research interests include open-loop control of hybrid dynamical systems, design and characterization of self-stabilizing mechanisms with applications in the legged locomotion, as well as design of embedded sensory and actuation systems for robotic platforms.

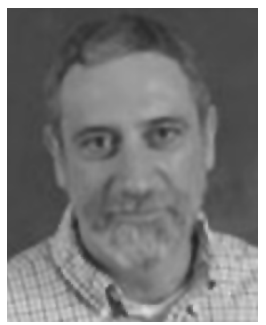

Daniel E. Koditschek (S'80-M'83-SM'93-F'04) received the $\mathrm{Ph} . \mathrm{D}$. degree in electrical engineering from Yale University, New Haven, CT, in 1983.

He was appointed to the faculty at Yale University in 1984 and moved to the University of Michigan, Ann Arbor, in 1993, where he is presently a Professor with the Department of Electrical Engineering and Computer Science with a joint appointment in the Artificial Intelligence Laboratory and Control Systems Laboratory. His research interests include robotics, the application of dynamical systems theory to intelligent mechanisms, nonlinear control, and applications of real-time distributed control technology.

Dr. Koditschek is a member of the American Association for the Advancement of Science, the Association for Computing Machinery, the American Mathematical Society, the Mathematical Association of America, the Society for Industrial and Applied Mathematics, and Sigma Xi. 